\title{
Innovation Qualité - La FMH mise sur la motivation
}

\section{Christoph Bosshard}

Dr méd., vice-président de la FMH, responsable du département Données, démographie et qualité et de l'ASQM

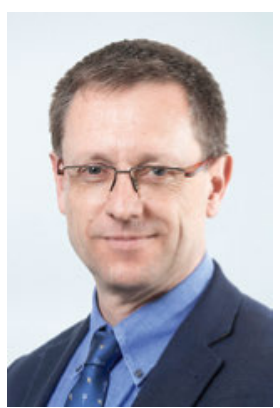

Mieux quelque chose fonctionne et moins nous en avons conscience, ce qui est logique en réalité. Avonsnous réellement conscience de tous les processus et structures qui se cachent derrière un horaire de transport public parfaitement rôdé? Il en va de même de notre santé: ce n'est qu'une fois malade que nous commençons à nous en soucier.

Et qu'en est-il de notre système de santé? En ce qui concerne sa qualité, différentes enquêtes internationales nous placent en tête de classement. Mais si l'on regarde le rapport coûts-PIB, nous occupons la même place que différents Etats européens que nous avons dépassés sur le plan de la qualité. Tout va donc pour le mieux? De quoi titiller notre fierté, même si nous savons que nous cessons d'être bons dès que nous cessons de chercher à nous améliorer.

Les sanctions ne sont pas un outil de gestion adapté: la qualité ne serait plus qu'un outil de comparaison, honnêteté et franchise appartiendraient au passé.

Cette approche ne doit cependant pas nous inciter à chercher des problèmes là où il n'y en a pas. L'excellent fonctionnement de notre système de santé, nous le devons à la forte motivation intrinsèque de tous les protagonistes. Et là où la motivation intrinsèque est si centrale, les objectifs et mécanismes de contrôle simplistes issus du fordisme sont voués à l'échec. Un système de santé complexe requiert des incitations un peu plus complexes. Un point qui n'a pas été suffisamment pris en compte jusqu'ici, ce qui explique pourquoi notre système de santé est si fortement marqué par les incitations négatives.

Faire reposer notre système de santé sur des sanctions n'aurait aucun sens. Un tel système aurait des effets collatéraux majeurs: la qualité ne serait plus qu'un outil de comparaison, honnêteté et franchise appartien- draient au passé, et toute chance de s'améliorer serait perdue. Mais surtout, les patients polymorbides seraient confrontés à un risque élevé de complication et seraient trimbalés d'un endroit à un autre telle une "patate chaude» au lieu d'être soignés.

Notre incitation intrinsèque pourrait se résumer ainsi: devenir meilleur ensemble et apprendre des erreurs ou simplement d'un moins bon résultat. La culture de l'amélioration continue représente donc un élément central des discussions au sein de l'Académie suisse pour la qualité en médecine (ASQM). Pour encourager les démarches qualité menées par nos organisations et nos partenaires et mieux les faire connaître, l'ASQM de la FMH a décidé de lancer le nouveau prix «Innovation Qualité», qui sera décerné pour la première fois en 2018. Vous en apprendrez davantage sur les différentes catégories de prix et les modalités d'inscription dans ce numéro du Bulletin des médecins suisses.

Saisissez l'occasion et participez à cette première édition en inscrivant votre projet en faveur de la qualité. Vous contribuerez ainsi à mettre en lumière des aspects fondamentaux du travail dans le domaine de la santé, ce qui est extrêmement précieux: trop souvent, ces «détails» essentiels restent invisibles en raison du parfait fonctionnement de notre système et ce n'est qu'une fois qu'ils ne sont plus là que nous prenons conscience de leur importance. De plus, en participant

\section{Inscrivez votre projet dès maintenant et} faites connaître votre démarche en faveur de la qualité dans le domaine de la santé!

à l'Innovation Qualité, vous donnez à d'autres la chance d'apprendre de votre projet et de vos expériences. Sans oublier, enfin, la belle récompense qui attend les lauréats. Seul celui qui ne participe pas n'a aucune chance de gagner! 